\title{
The effect of PD-L1/PD-1 immunotherapy in the treatment of squamous non-small-cell lung cancer: a meta-analysis of randomized controlled clinical trials
}

\author{
Shuang Li, Shuang Zhang, Jingjing Liu, Changliang Yang, Liang Zhang, Ying Cheng \\ Department of Thoracic Oncology, Jilin Cancer Hospital, Changchun 130012, China \\ Contributions: (I) Conception and design: Y Cheng, S Li; (II) Administrative support: Y Cheng; (III) Provision of study materials or patients: C Yang, \\ L Zhang; (IV) Collection and assembly of data: S Li, S Zhang, J Liu; (V) Data analysis and interpretation: Y Cheng, S Li; (VI) Manuscript writing: \\ All authors; (VII) Final approval of manuscript: All authors. \\ Correspondence to: Ying Cheng. Department of Medical Oncology, Jilin Cancer Hospital, No. 1018, Huguang Road, Chaoyang Borough, Changchun \\ 130012, China. Email: chengying@csco.org.cn.
}

Background: This meta-analysis evaluated the efficacy of PD-L1/PD-1 immunotherapy compared with chemotherapy in IIIB or IV or recurrent squamous non-small cell lung cancer (NSCLC) patients.

Methods: We performed a literature search on the PubMed, EMBASE, Web of Science, and Cochrane databases, in addition to the abstracts from major conference proceedings of the European Society for Medical Oncology (ESMO), the American Society of Clinical Oncology (ASCO), and the World Conference on Lung Cancer (WCLC) from 2004 to May 2019. Randomized controlled trials that compared PD-L1/ PD-1 immunotherapy with chemotherapy in IIIB or IV or recurrent squamous NSCLC were included. The endpoints were overall survival (OS), progression-free survival (PFS), objective response rate (ORR), disease control rate (DCR), 1-y OS rate, and 1-y PFS rate.

Results: A total of 3,213 IIIB or IV or recurrent squamous NSCLC patients from 12 trials of high quality were included in this meta-analysis, among whom 1,677 were in the PD-L1/PD-1 immunotherapy group and 1,536 were in the chemotherapy group. The results indicated that compared with chemotherapy, immunotherapy significantly prolonged OS (HR =0.75; 95\% CI, 0.68-0.83; $\mathrm{P}<0.001)$ and PFS (HR $=0.66 ; 95 \% \mathrm{CI}, 0.60-0.73 ; \mathrm{P}<0.001)$ in patients with advanced squamous NSCLC. The pooled model showed that ORR, DCR, and 1-y PFS rate were also significantly higher with immunotherapy compared with chemotherapy (ORR: RR =1.43; 95\% CI, 1.11-1.83; P=0.005; DCR: RR =1.14; 95\% CI, 1.05-1.24; $\mathrm{P}=0.003 ; 1-y$ PFS rate: $\mathrm{RR}=2.30 ; 95 \% \mathrm{CI}, 1.69-3.13$; $\mathrm{P}<0.001)$. The 1-y OS rate tended to be higher in the immunotherapy group, but there was no significant difference compared with chemotherapy $(\mathrm{RR}=1.28 ; 95 \%$ CI, 0.95-1.72; P=0.104).

Conclusions: Our study proves that PD-L1/PD-1 immunotherapy can significantly improve OS, PFS, ORR, DCR, and 1-y PFS of metastatic squamous NSCLC patients compared with chemotherapy.

Keywords: Programmed death-1 receptor inhibitor; immunotherapy; squamous; non-small cell lung cancer (NSCLC)

Submitted Jul 10, 2019. Accepted for publication Oct 17, 2019.

doi: $10.21037 /$ jtd.2019.11.12

View this article at: http://dx.doi.org/10.21037/jtd.2019.11.12

(c) Journal of Thoracic Disease. All rights reserved. 


\section{Introduction}

Cancer is a major public health problem worldwide, and in 2015, at least 1.69 million people worldwide died from lung cancer $(1,2)$. Non-small cell lung cancer (NSCLC) accounts for the majority of lung cancer and can be subdivided into adenocarcinoma, squamous cell carcinoma, adenosquamous cell carcinoma, large cell carcinoma, sarcomatoid carcinoma, and lung squamous cell carcinoma, with the latter accounting for about $30-40 \%$ (2) of NSCLC, $90 \%$ of which is related to smoking (3-6).

NSCLC treatment has entered the era of precision therapy, and targeted therapy, such as epidermal growth factor receptor-tyrosine kinase inhibitor (EGFR-TKI) has been very successful for driver gene-positive lung adenocarcinoma (7-9). Although recent progress has been made understanding the driving mutations in lung squamous cell carcinoma, there have still been no major breakthroughs in targeted drugs in lung squamous cell carcinoma. The treatment of squamous NSCLC is still stalled in chemotherapy, with a low efficacy rate of only about $30 \%$, and the survival time of patients with advanced squamous NSCLC is only about one year (10-14). Treatment for advanced squamous NSCLC remains an unmet need.

Immunotherapy can kill and control tumors by enhancing or restoring the anti-tumor immune function. PD-1/PD-L1 exerts immunosuppressive function in vivo and plays an important role in the process of tumor immune escape. PD-1 is normally expressed on T cells, and its ligands PD-L1 and PD-L2 are often expressed on tumor cells and infiltrating immune cells. PD-L and PD-L1 inhibitors can enhance the anti-tumor ability of the immune system by inhibiting the binding of PD-1 and PD-L1 $(15,16)$. Recently, PD-1/PD-L1 inhibitors have made breakthroughs in the treatment of various tumors, including NSCLC. Most studies on PD-1/PDL1 inhibitors in NSCLC have included squamous cell carcinoma and adenocarcinoma at the same time; however, immunotherapy specifically for squamous cell lung cancer is very limited. Among the treatments of advanced NSCLC, only the CheckMate 063 (17) and CheckMate 017 studies were designed specifically to investigate lung squamous cell carcinoma, and only the KEYNOTE407 and IMpower131 studies have been conducted for the immunotherapy of advanced primary lung squamous cell carcinoma.

In the CheckMate 017 study (18), the clinical benefit of treatment with nivolumab was significant for advanced squamous-cell NSCLC, and overall survival (OS) was prolonged by 3.2 month $(\mathrm{m})$ compared with docetaxel. The KEYNOTE 407 study (19) and IMpower131 study (20) reported at the 2018 American Society of Clinical Oncology (ASCO) Annual Meeting, were similarly phase 3 studies and focused on first-line treatment of advanced squamous NSCLC, but the results were inconsistent. The KEYNOTE 407 study showed that pembrolizumab plus chemotherapy could benefit patients with advanced squamous NSCLC on OS, while the IMpower 131 interim analysis for OS suggested that there was no statistical difference between the atezolizumab plus chemotherapy group and the chemotherapy group for patients with advanced squamous NSCLC. Although advanced lung squamous cell carcinoma patients could benefit from immunotherapy in these studies, more evidence is needed to support immunotherapy for lung squamous cell carcinoma.

Based on the background described above, we conducted a meta-analysis that included a large sample of IIIB or IV or recurrent squamous NSCLC patients to explore whether PD-L1/PD-1 immunotherapy has therapeutic advantages in squamous NSCLC compared to chemotherapy.

\section{Methods}

\section{Search method}

We performed a literature search on the PubMed, EMBASE, Web of Science, and Cochrane databases, in addition to the abstracts from major conference proceedings of the European Society for Medical Oncology (ESMO), the American Society of Clinical Oncology (ASCO), and World the Conference on Lung Cancer (WCLC) from 2004 to May 2019. All published randomized trials comparing PD-L1/PD-1 immunotherapy versus chemotherapy of IIIB or IV or recurrent squamous NSCLC were identified. The keywords used were as follows: "squamous", "non-smallcell lung cancer OR non-small-cell lung carcinoma OR NSCLC" "immunotherapy", "PD-L1/PD-1", "nivolumab", "pembrolizumab", "atezolizumab", "randomized", and "phase II study OR phase III study". In addition to this, the reference lists of included clinical trials were manually screened.

\section{Inclusion criteria}

The inclusion criteria were as follows:

(I) studies were phase II or III randomized controlled 
trials;

(II) studies were designed as parallel-arm to compare PD-L1/PD-1 immunotherapy versus chemotherapy;

(III) patients were confirmed squamous NSCLC by histopathology;

(IV) studies provided one of OS, progression-free survival (PFS), objective response rate (ORR), disease control rate (DCR), or the information which could calculate them;

(V) studies included full text with original data, while the abstracts from major conference proceedings of ESMO, ASCO, and WCLC provided sufficient information on study design and outcomes;

(VI) when duplicate studies appeared, we included the newest, larger sample size, or the study of higher quality.

\section{Data extraction}

Two reviewers (S Li and S Zhang), after carefully reading the full text of included literature, abstracted data independently in accordance with the Preferred Reporting Items for Systematic Reviews and Meta-analyses statement (21). If there were any discrepancies between the two reviewers during the process, a third reviewer was involved in resolving the issue by consensus. The following data were collected from each article: name of study, year of publication, phase of study, patient characteristics, treatment comparison, cases of squamous NSCLC of treatment group and control group, and HRs with 95\% CIs for OS, PFS, ORR, DCR, 1-y OS rate, and 1-y PFS rate. The risk of bias of the trials was assessed by using the risk of bias tool of The Cochrane Collaboration.

\section{Statistical analysis}

This meta-analysis was completed using STATA V.12.0 (Stata Corporation, College Station, TX, USA). HR and $95 \%$ CI were calculated as the overall effect for OS and PFS. An HR $<1.0$ suggested that the treatment effect for PD-L1/PD-1 immunotherapy was superior to chemotherapy. The relative risk (RR) was calculated as an effective measure for ORR, DCR, 1-y OS rate, and 1-y $\mathrm{PFS}$ rate. $\mathrm{P}$ value of $\mathrm{Q}$ test and $\mathrm{I}^{2}$ was used to evaluate the statistical heterogeneity. If $\mathrm{I}^{2}>50 \%$ and $\mathrm{P}$ value $<0.1$, we considered heterogeneity to be present, and a randomeffects model was adopted. If heterogeneity was not discovered, a fixed-effects model was adopted. Publication biases were tested by Begg's funnel plot and Egger's test. A two-sided $\mathrm{P}$ value of $<0.05$ was considered statistically significant for results.

\section{Results}

In accordance with our search strategies, searching was first conducted with the preliminary screening, and, after excluding reviews, single-arm designed studies, and irrelevant research, 12 trials of high quality were included in our meta-analysis. A total of 3,213 IIIB or IV or recurrent squamous NSCLC patients were included, among whom 1,677 were in the PD-L1/PD-1 immunotherapy group, and 1,536 were in the chemotherapy group. Of the 12 studies, 4 studies compared nivolumab/nivolumab + ipilimumab $v s$. chemotherapy, 4 studies compared pembrolizumab/ pembrolizumab + chemotherapy $v s$. chemotherapy, 3 studies compared atezolizumab $v s$. chemotherapy, and 1 study compared avelumab vs. chemotherapy.

Tumor cells were used to measure PD-L1 expression in evaluation trials of nivolumab, pembrolizumab, and avelumab, while tumor cells and/or immune cells were used to measured PD-L1 expression in the atezolizumab trials.

The process of screening is shown in a flow chart (Figure 1). The baseline characteristics of the 4 studies are displayed in Table 1. The risk of bias assessment is shown in Figure 2.

\section{OS}

Data for OS were available from 10 included studies (18-20,22,23,25,26,28-30). A fixed effects model was used since the heterogeneity test suggested that there was no heterogeneity between studies $\left(\mathrm{I}^{2}=18.4 \%, \mathrm{P}=0.273\right)$. The results showed that compared with chemotherapy, immunotherapy significantly prolonged OS in patients with advanced squamous NSCLC (HR $=0.75$; $95 \%$ CI, $0.68-$ 0.83; $\mathrm{P}<0.001$ ) (Figure $3 A$ ).

Three trials reported 1-y OS rate data (18-20). For 1-y OS, no statistically significant difference was observed between the immunotherapy and chemotherapy groups $(\mathrm{RR}=1.28 ; 95 \%$ CI, 0.95-1.72; $\mathrm{P}=0.104)$. A random effects model was used since heterogeneity across the trials was significant $\left(\mathrm{I}^{2}=86.9 \%\right.$, $\mathrm{P}<0.001$ ) (Figure S1). The 2-y OS rate was exploratory analyzed, and got the same conclusion (Figure S2).

\section{Subgroup analyses of $O S$}

Subgroup analyses were conducted according to the line of 


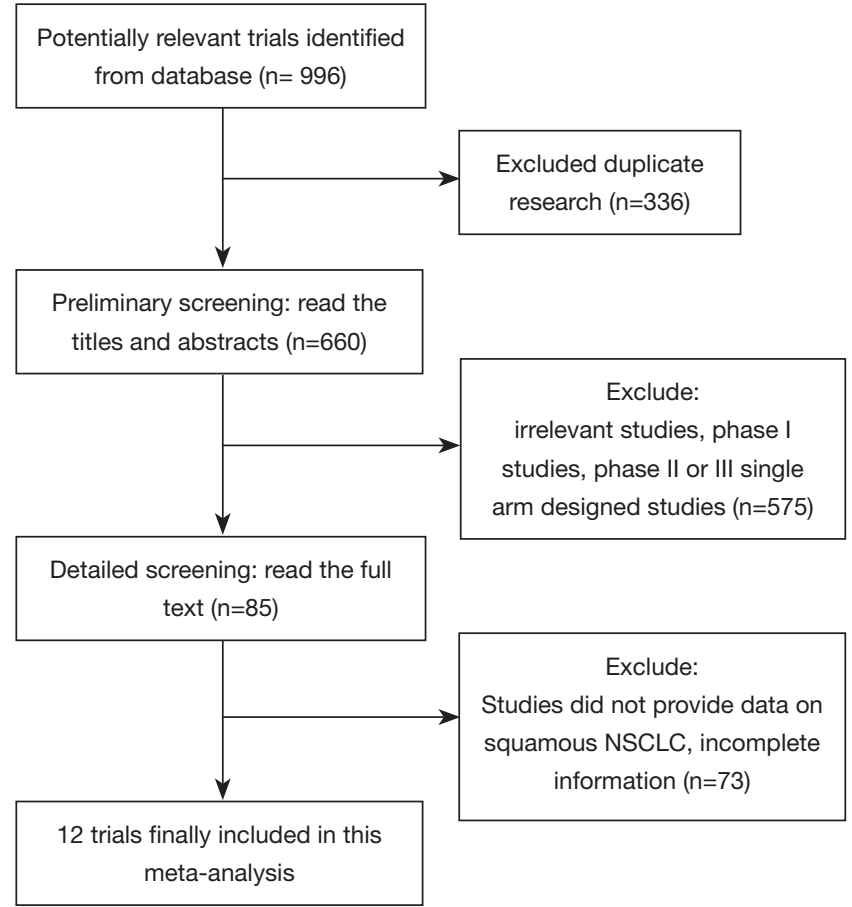

Figure 1 Flow chart of trial selection process

treatment, PD-L1 expression level, and experimental drug; the trends of treatment effects were similar between the subgroups.

Four trials reported HRs for OS of immunotherapy used as first-line treatment, and 6 trials reported those for second-line treatment and above. Overall, the pooled hazard ratio showed that regardless of being used as first-line [HR: $0.81(0.72-0.92), \mathrm{P}=0.001]$ or second-line and above [HR: 0.68 (0.59-0.78), $\mathrm{P}<0.001]$ treatment, immunotherapy was superior to chemotherapy in OS (Figure $3 B$ ).

Six trials included all-comer patients, and 4 trials included patients with $\mathrm{PD}-\mathrm{L} 1 \geq 1 \%$. As shown in the forest plot, it can be seen that both in all-comer [HR: 0.74 (0.66-0.84), $\mathrm{P}<0.001]$ and in PD-L1 $\geq 1 \%$ [HR:0.77 (0.66$0.89), \mathrm{P}=0.001$ ] patients, immunotherapy was superior to chemotherapy in OS (Figure 3C).

For 6 trials, the treatment group was PD-1 inhibitor, and for 4 trials, it was PD-L1 inhibitor. The pooled HRs for OS showed that, whether PD-1 inhibitor [HR: $0.70(0.62-0.79)$, $\mathrm{P}<0.001$ ] or PD-L1 inhibitor [HR: 0.84 (0.73-0.97), $\mathrm{P}=0.021$ ] treatment, there was significant improvement in OS for immunotherapy compared with chemotherapy (Figure 3D).

\section{PFS}

Data for PFS were available from 8 included studies (18-
20,23,24,26-28). A fixed-effects model was used since the heterogeneity test suggested that there was no heterogeneity between studies $\left(\mathrm{I}^{2}=27.5 \%, \mathrm{P}=0.209\right)$. The results showed a significantly longer PFS with immunotherapy than with chemotherapy in patients with advanced squamous NSCLC (HR $=0.66 ; 95 \% \mathrm{CI}, 0.60-0.73 ; \mathrm{P}<0.001$ ) (Figure 4A).

Two trials reported $1-y$ PFS rate data $(18,20)$. The pooled model showed that 1-y PFS was also significantly higher with immunotherapy compared with chemotherapy $(\mathrm{RR}=2.30 ; 95 \% \mathrm{CI}, 1.69-3.13 ; \mathrm{P}<0.001)$. A fixedeffects model was applied due to the lack of significant heterogeneity $\left(\mathrm{I}^{2}=41.5 \%, \mathrm{P}=0.191\right)$ (Figure S3).

\section{Subgroup analyses of PFS}

Five trials reported HRs for PFS of immunotherapy used as first-line treatment, and 3 trials reported those for secondline treatment and above. Overall, the pooled hazard ratio showed that, regardless of being used as first-line [HR: $0.65(0.57-0.73) ; \mathrm{P}<0.001]$ or second-line and above [HR: 0.69 (0.58-0.83); $\mathrm{P}<0.001]$ treatment, immunotherapy was superior to chemotherapy in PFS (Figure $4 B$ ).

Four trials included all-comer patients, 3 trials included patients with $\mathrm{PD}-\mathrm{L} 1 \geq 1 \%$, and 1 trial included patients with $\mathrm{PD}-\mathrm{L} 1 \geq 50 \%$. As shown in the forest plot, it can be seen that regardless of being all-comer [HR: $0.64(0.57-0.72)$; $\mathrm{P}<0.001$ ], $\mathrm{PD}-\mathrm{L} 1 \geq 1 \%$ [HR: 0.78 (0.63-0.97); $\mathrm{P}=0.024$, or PD-L1 $\geq 50 \%$ patients [HR: 0.35 (0.17-0.72); $\mathrm{P}=0.004$ ], immunotherapy was superior to chemotherapy in PFS (Figure 4C).

For 6 trials, the treatment group was PD-1 inhibitor, and for 2 trials, it was PD-L1 inhibitor. The pooled HRs for PFS showed that, whether PD-1 inhibitor [HR: 0.64 (0.56$0.73), \mathrm{P}<0.001]$ or PD-L1 inhibitor [HR: $0.70(0.60-0.82)$, $\mathrm{P}<0.001]$ treatment, there was significant improvement in PFS for immunotherapy compared with chemotherapy (Figure 4D).

\section{ORR and DCR}

Three trials reported ORR data (18-20). The pooled model showed that ORR was also significantly higher with immunotherapy compared with chemotherapy $(\mathrm{RR}=1.43$; 95\% CI, 1.11-1.83; $\mathrm{P}=0.005)$. A random effects model was used since heterogeneity across the trials was significant $\left(\mathrm{I}^{2}=67.3 \%, \mathrm{P}=0.047\right)$ (Figure S4).

Two trials reported DCR data $(18,19)$. The pooled model showed that DCR was also significantly higher with 


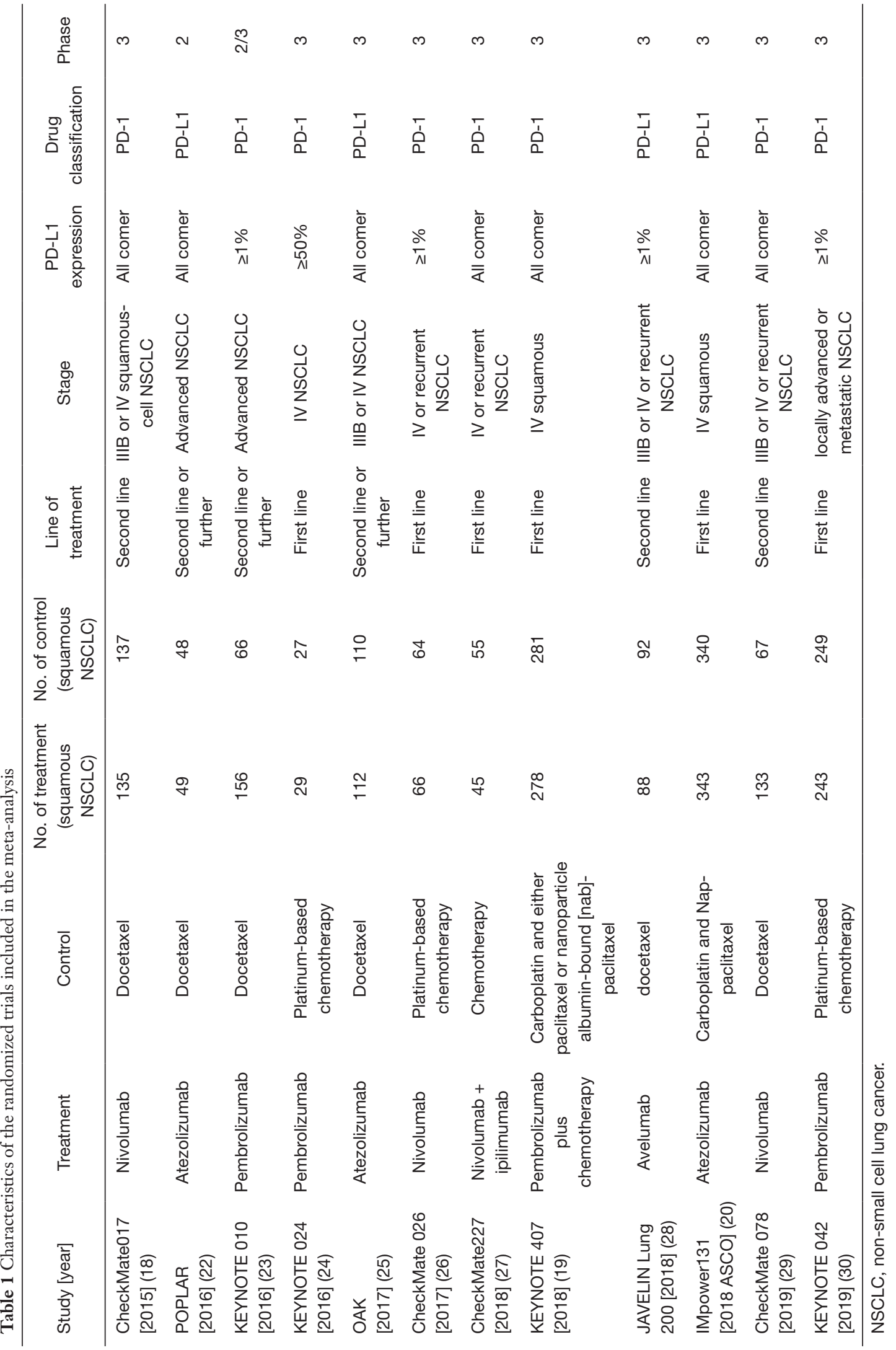




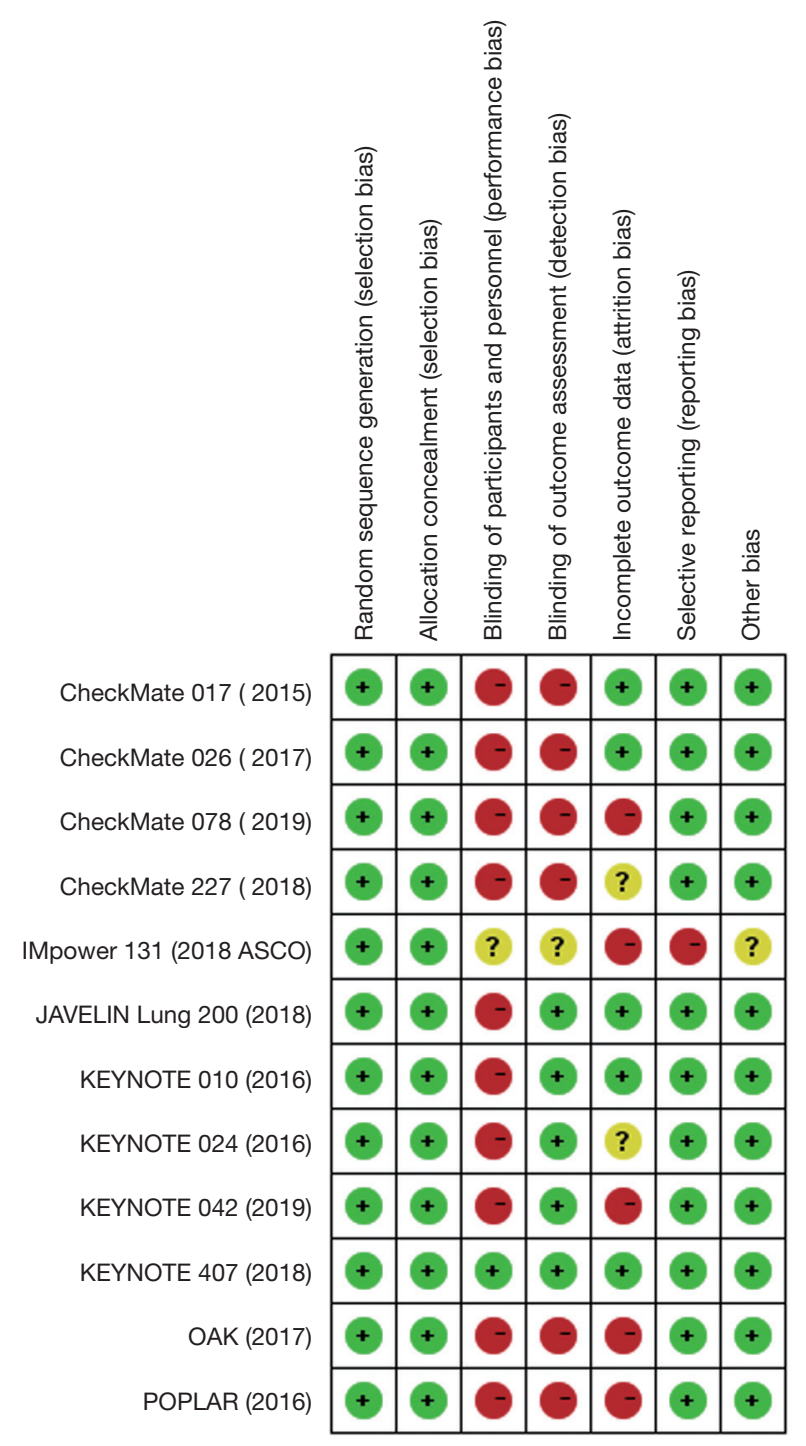

Figure 2 Risk of bias summary of included trials.

immunotherapy compared with chemotherapy $(\mathrm{RR}=1.14$; 95\% CI, 1.05-1.24; $\mathrm{P}=0.003)$. A fixed effects model was applied due to a lack of significant heterogeneity $\left(\mathrm{I}^{2}=0 \%\right.$, $\mathrm{P}=0.976$ ) (Figure S5).

\section{Publication bias and sensitivity analysis}

Begg's funnel plot for OS and PFS did not suggest any evidence of obvious asymmetry, and the Egger's test results showed that there was no publication bias $(\mathrm{P}=0.721$ for OS; $\mathrm{P}=0.711$ for $\mathrm{PFS}$ ). Sensitivity analysis indicated that the results of this meta-analysis were not affected by the exclusion of a particular trial from the analysis.

\section{Discussion}

Our study is, to date, the most comprehensive meta-analysis of its type: we reviewed all the published clinical trials of high quality and clinical significance that were related to PD-L1/PD-1 immunotherapy versus chemotherapy of IIIB or IV or recurrent squamous NSCLC.

Immunotherapy, especially immune checkpoint inhibitors (ICIs) of lung cancer, has been successfully used in the treatment of lung cancer. Its use breaks the traditional concept that melanoma and renal cancer are uniquely "immunogenic" cancers. It has also provided a new approach for the development of immunotherapy for other cancers (31).

The main mechanism of ICIs is to kill tumor cells by activating the autoimmune immune system of tumor patients by removing the immunosuppressive signal of $\mathrm{T}$ cells. As a member of the immunoglobulin superfamily, PD-1 is expressed on immune cells, including T cells and $\mathrm{B}$ cells, and participates in the transmission of inhibitory signals through ligands PD-L1 and PD-L2. PD-L1 is the main ligand of PD-1, the activation of the $\mathrm{PD}-1 / \mathrm{PD}-\mathrm{L} 1$ pathway is conducive to tumor immune escape, and blocking the PD-1/PD-L1 pathway can enhance endogenous anti-tumor immunity (32-36).

This meta-analysis combined 3213 patients from 12 randomized trials. Our study highlighted that, compared with chemotherapy, PD-1/PD-L1 immunotherapy could reduce risk of death by $25 \%$ and risk of progression by $34 \%$ for IIIB or IV or recurrent squamous NSCLC patients. ORR, DCR, and 1-y PFS rates were also increased. The heterogeneity tests of the pooled results showed that there was no significant statistical heterogeneity in the included trials. Therefore, the pooled results of this study have a certain clinical guiding significance. This conclusion has been confirmed in many large phase III multicenter randomized controlled studies $(18,19,25,29,30)$. CheckMate 017 study (18) and CheckMate 078 study (29) both explored the efficacy of nivolumab in second-line and above treatments in advanced squamous NSCLC, and the results showed that nivolumab could reduce the risk of death by nearly $40 \%$ compared with chemotherapy. The subgroup analysis of the OAK study (25) showed that compared with docetaxel, the second-line treatment of atezolizumab could significantly prolong the OS (HR $=0.73$; median OS: 8.9 vs. $7.7 \mathrm{~m}$ ) for advanced squamous NSCLC patients. The KEYNOTE 407 study (19) further explored the efficacy of pembrolizumab combined with chemotherapy in first-line 


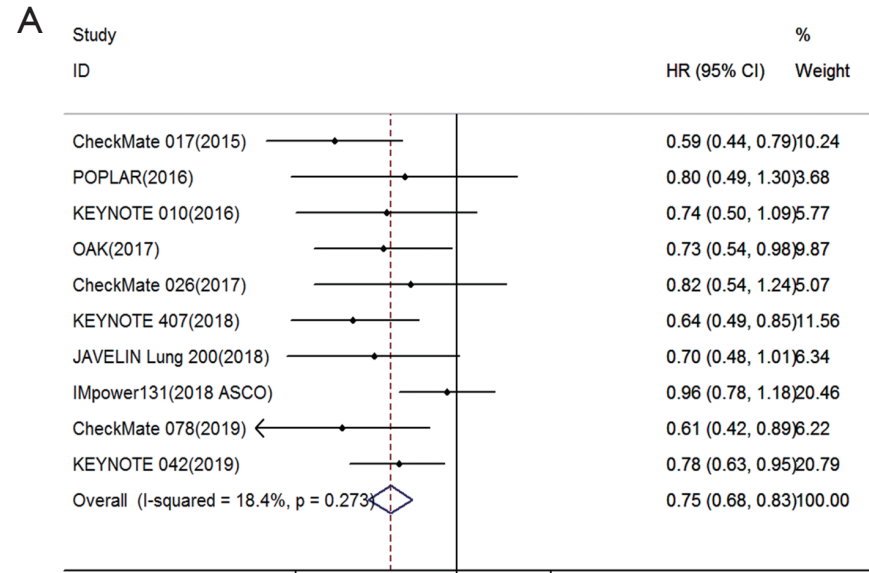

C

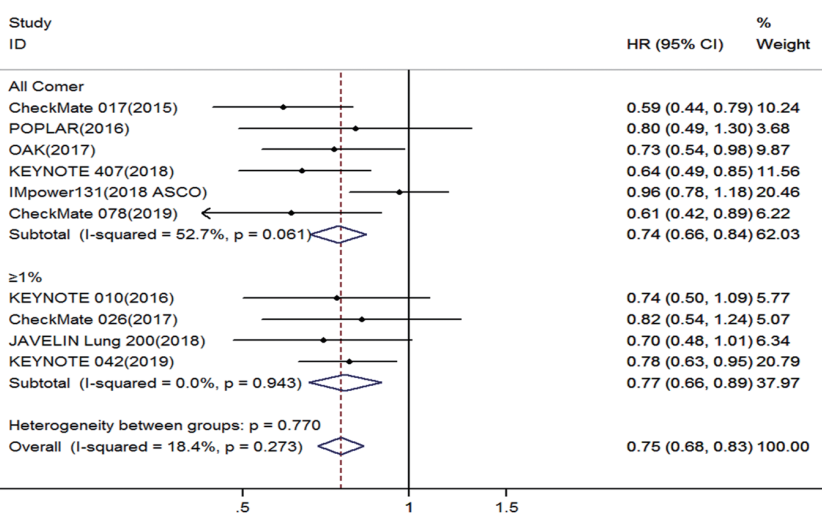

B

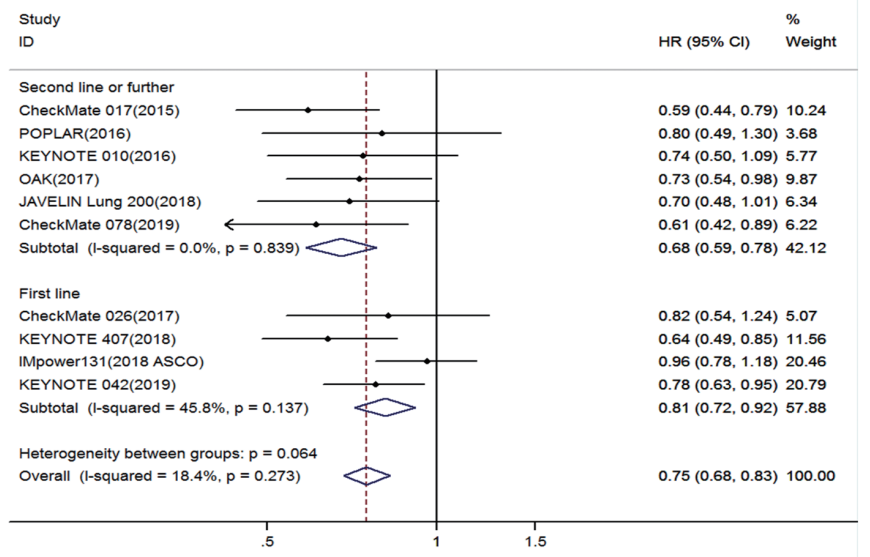

D

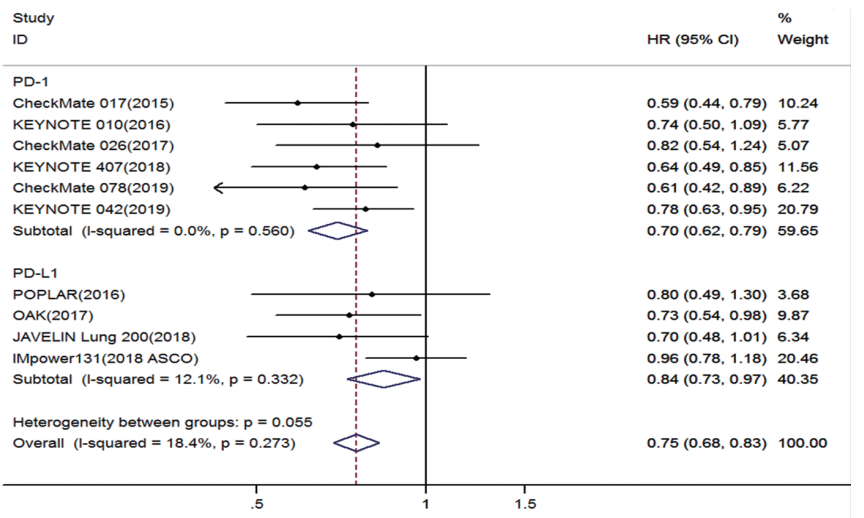

Figure 3 Forest plot of overall survival for comparison of PD-1/PD-L1 immunotherapy to chemotherapy in squamous non-small cell lung cancer (A); subgroup of the line of treatment (B); subgroup of PD-L1 expression level (C); subgroup of experimental drug (D).

treatment of advanced squamous NSCLC. A total of 559 primary metastatic squamous NSCLC patients were enrolled in this study, and the results showed that pembrolizumab combined with chemotherapy significantly prolonged PFS (median PFS: 6.4 vs. $4.8 \mathrm{~m}$; HR =0.56) and OS (median PFS: 15.9 vs. $11.3 \mathrm{~m} ; \mathrm{HR}=0.64$ ). Subgroup analysis showed that both PD-L1 positive and PD-L1 negative patients could benefit from immune combination therapy.

It can be seen from our meta-analysis that the improvement of OS and PFS can be achieved compared with chemotherapy whether immunotherapy is received from the first-line or second-line treatment. We found that the HR of second-line immunotherapy was 0.68 , and that of the first-line immunotherapy was 0.81 . This result also prompted us to consider when immunotherapy was better for interventional therapy in lung squamous cell carcinoma. In the first-line immunotherapy studies we included, some patients in the chemotherapy group received subsequent immunotherapy. In the KEYNOTE407 study (19) design, the chemotherapy group allowed subsequent cross-over to the immunotherapy group, and a total of $31.7 \%$ of patients in the chemotherapy group received subsequent immunotherapy, including 75 cases in the study, and 14 cases outside the study. Similarly, $64.2 \%$ of patients in the chemotherapy group in the KEYNOTE 024 study (24) received subsequent immunotherapy, while this occurred in $20 \%$ of patients in the KEYNOTE 042 study (30), $42.1 \%$ in the IMpower 131 study, and $60.4 \%$ in the CheckMate 026 study. It is suggested that immunotherapy from the first line has a better prognosis in squamous cell lung cancer. In addition, the KEYNOTE189 study (37) was based on the study of lung adenocarcinoma (ASCO 2019). Progression from randomized to second-line treatment (PFS2) was significantly better in the immunotherapy group than in the chemotherapy group (17 vs. 9 mo). As seen from the results of PFS2, the efficacy of first-line immunotherapy is 
A

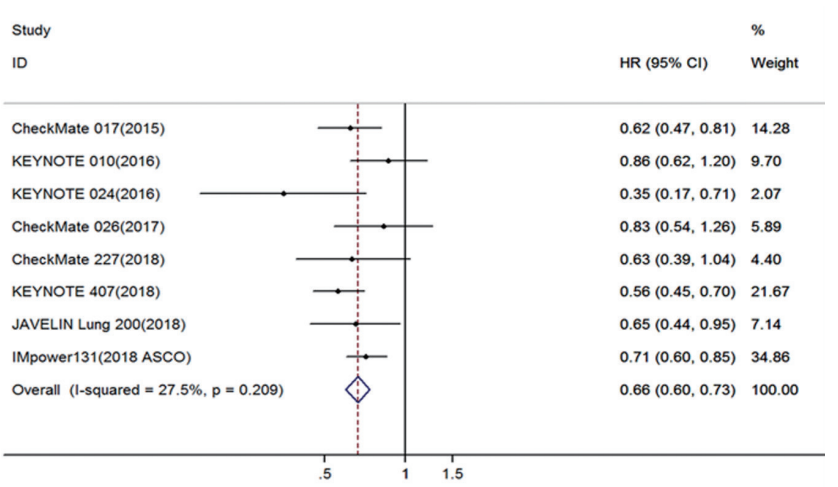

C

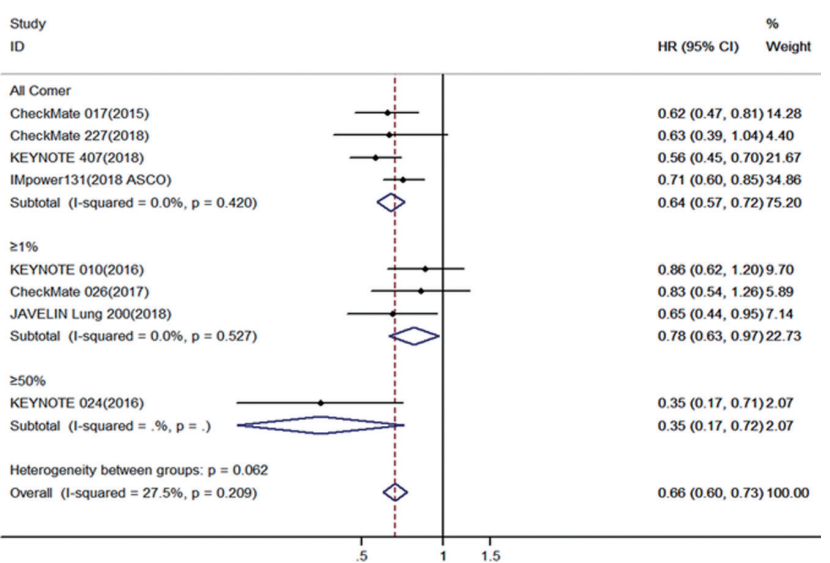

B

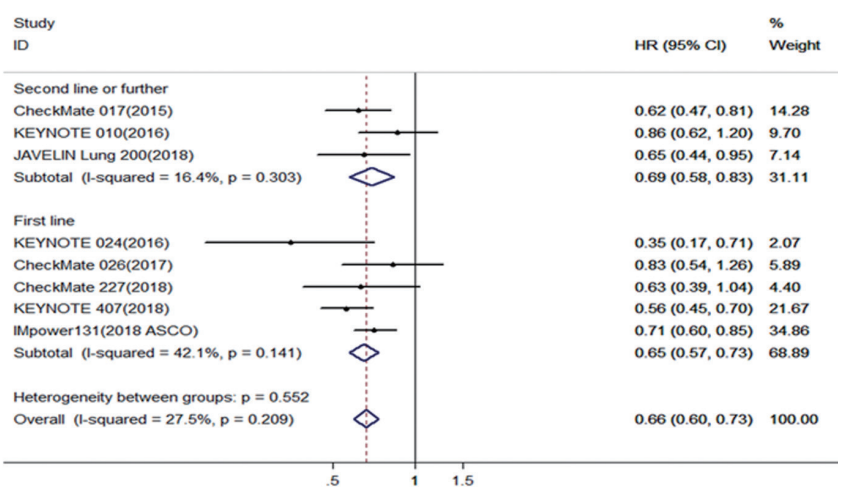

D

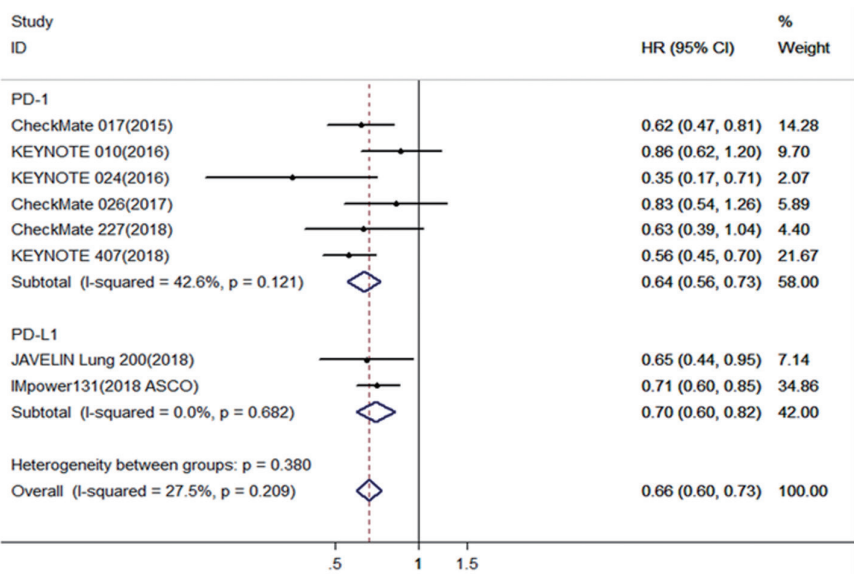

Figure 4 Forest plot of progression-free survival for comparison of PD-1/PD-L1 immunotherapy to chemotherapy in squamous non-small cell lung cancer (A); subgroup of line of treatment (B); subgroup of PD-L1 expression level (C); subgroup of experimental drug (D).

significantly better than that of second-line immunotherapy, suggesting that patients who use first-line immunotherapy as early as possible will benefit more. Of course, forwardlooking research is needed to verify this claim.

The question of which strategy is better, immune combination or immune monotherapy, is also a hot topic in immunotherapy. In the studies we included, secondline treatment was based on immune monotherapy, firstline treatment with CheckMate 026, and KEYNOTE 024/042 also used monotherapy, while KEYNOTE407 and IMpower131 used immunotherapy combined with chemotherapy. Some chemotherapeutic drugs can promote the release of a large number of antigens in tumor cells while increasing antigen presentation, and chemotherapy, combined synergistically with immunotherapy, can also remove some immunosuppressive cells. A meta-analysis (38) published in 2019 included the KEYNOTE-021, KEYNOTE-189, KEYNOTE-407, KEYNOTE-024, and KEYNOTE-042 studies, with the results suggesting that for advanced NSCLC patients, both pembrolizumab plus chemotherapy (PFS: HR 0.36; OS: HR 0.51) and pembrolizumab alone (PFS: HR, 0.65; OS: HR 0.67) improved clinical outcomes compared with chemotherapy as first line treatment. We did not conduct this type of analysis in our study, as there were many confounding factors, including a limited number of eligible combined immunotherapy studies, the inclusion of PD-1 and PD-L1 inhibitors, and the inclusion of first and second-line immune-monotherapy. With the release of more research data, we will further explore this aspect in the future and provide more evidence for clinical treatment options.

PD-L1 is an established biomarker for selecting patients for treatment with immunotherapy (24). Our research found that prolongations of OS and PFS of a consistent magnitude were observed across the categories of PD-L1 tumor proportion score. PFS with $\mathrm{PD}-\mathrm{L} 1 \geq 50 \%$ was more likely to 
benefit than PD-L1 $\geq 1 \%$ or all-comers (for $\geq 50 \%$ : HR $=0.35$; for $\geq 1 \%$ : $H R=0.78$; for all comer: $H R=0.64)$. Meanwhile, the OS of patients with PD-L $1 \geq 1 \%$ or all-comers showed similar benefits. Keynote 024 (24), a randomized controlled phase III study, included 56 primary advanced squamous NSCLC patients with PD-L $1 \geq 50 \%$ and without the EGFR or ALK mutation. Compared with the chemotherapy group, pembrolizumab significantly reduced the risk of disease progression by $65 \%$. The Keynote 042 study (39) further expanded the inclusion criteria to $\mathrm{PD}-\mathrm{L} 1 \geq 1 \%$ : the study demonstrated that pembrolizumab reduced the risk of death in patients with advanced squamous NSCLC by $22 \%$ compared with chemotherapy. Subgroup analysis also suggested that the main beneficiaries were patients with $\mathrm{PD}-\mathrm{L} 1 \geq 50 \%$.

Despite its insightful findings, this meta-analysis has several limitations. Firstly, the IMpower131 study was only available through ASCO abstracts and has not yet been published. Secondly, not all included studies were specifically designed for squamous NSCLC patients; therefore, most results were extracted from subgroup analyses for pathological subtypes. Thirdly, not all studies reported OS, PFS, ORR, DCR, 1-y OS rate, or 1-y PFS rate. Therefore, the results should be interpreted with caution. More prospective phase III randomized controlled studies should be carried out in the future to explore the efficacy and safety of PD-L1/PD-1 immunotherapy for advanced squamous NSCLC, providing strong clinical evidence for squamous NSCLC to receive ICI therapy.

\section{Conclusions}

Our study proved that PD-L1/PD-1 immunotherapy could significantly improve the OS, PFS, ORR, DCR, and 1-y PFS rate of advanced squamous NSCLC patients compared with chemotherapy, but further evaluation and verification are required for future phase III studies.

\section{Acknowledgments}

Funding: This work was supported by the Health and Family Planning Commission of Jilin Province (No. 2018W008).

\section{Footnote}

Conflicts of Interest: The authors have no conflicts of interest to declare.
Ethical Statement: The authors are accountable for all aspects of the work in ensuring that questions related to the accuracy or integrity of any part of the work are appropriately investigated and resolved.

\section{References}

1. Siegel RL, Miller KD, Jemal A. Cancer statistics, 2019. CA Cancer J Clin 2019;69:7-34.

2. Hammerschmidt $\mathrm{S}$, Wirtz H. Lung cancer: current diagnosis and treatment. Dtsch Arztebl Int 2009;106:80918; quiz 819-20.

3. Lortet-Tieulent J, Soerjomataram I, Ferlay J, et al. International trends in lung cancer incidence by histological subtype: adenocarcinoma stabilizing in men but still increasing in women. Lung Cancer 2014;84:13-22.

4. Cheng TY, Cramb SM, Baade PD, et al. The International Epidemiology of Lung Cancer: Latest Trends, Disparities, and Tumor Characteristics. J Thorac Oncol 2016;11:1653-71.

5. Soldera SV, Leighl NB. Update on the Treatment of Metastatic Squamous Non-Small Cell Lung Cancer in New Era of Personalized Medicine. Front Oncol 2017;7:50.

6. Socinski MA, Obasaju C, Gandara D, et al. Current and Emergent Therapy Options for Advanced Squamous Cell Lung Cancer. J Thorac Oncol 2018;13:165-83.

7. Zhou C, Wu YL, Chen G, et al. Erlotinib versus chemotherapy as first-line treatment for patients with advanced EGFR mutation-positive non-small-cell lung cancer (OPTIMAL, CTONG-0802): a multicentre, open-label, randomised, phase 3 study. Lancet Oncol 2011;12:735-42.

8. Cheng Y, Murakami H, Yang PC, et al. Randomized Phase II Trial of Gefitinib With and Without Pemetrexed as First-Line Therapy in Patients With Advanced Nonsquamous Non-Small-Cell Lung Cancer With Activating Epidermal Growth Factor Receptor Mutations. J Clin Oncol 2016;34:3258-66.

9. Fukuoka $\mathrm{M}, \mathrm{Wu} \mathrm{YL}$, Thongprasert S, et al. Biomarker analyses and final overall survival results from a phase III, randomized, open-label, first-line study of gefitinib versus carboplatin/paclitaxel in clinically selected patients with advanced non-small-cell lung cancer in Asia (IPASS). J Clin Oncol 2011;29:2866-74.

10. Ohe Y, Ohashi Y, Kubota K, et al. Randomized phase III study of cisplatin plus irinotecan versus carboplatin plus paclitaxel, cisplatin plus gemcitabine, and cisplatin 
plus vinorelbine for advanced non-small-cell lung cancer: Four-Arm Cooperative Study in Japan. Ann Oncol 2007;18:317-23.

11. Scagliotti GV, Parikh P, von Pawel J, et al. Phase III study comparing cisplatin plus gemcitabine with cisplatin plus pemetrexed in chemotherapy-naive patients with advanced-stage non-small-cell lung cancer. J Clin Oncol 2008;26:3543-51.

12. Danson S, Middleton MR, O'Byrne KJ, et al. Phase III trial of gemcitabine and carboplatin versus mitomycin, ifosfamide, and cisplatin or mitomycin, vinblastine, and cisplatin in patients with advanced nonsmall cell lung carcinoma. Cancer 2003;98:542-53.

13. Fossella F, Pereira JR, von Pawel J, et al. Randomized, multinational, phase III study of docetaxel plus platinum combinations versus vinorelbine plus cisplatin for advanced non-small-cell lung cancer: the TAX 326 study group. J Clin Oncol 2003;21:3016-24.

14. Schiller JH, Harrington D, Belani CP, et al. Comparison of four chemotherapy regimens for advanced non-smallcell lung cancer. N Engl J Med 2002;346:92-8.

15. Chen YB, Mu CY, Huang JA. Clinical significance of programmed death-1 ligand-1 expression in patients with nonsmall cell lung cancer: a 5-year-follow-up study. Tumori 2012;98:751-5.

16. Velcheti V, Schalper KA, Carvajal DE, et al. Programmed death ligand-1 expression in non-small cell lung cancer. Lab Invest 2014;94:107-16.

17. Rizvi NA, Mazières J, Planchard D, et al. Activity and safety of nivolumab, an anti-PD-1 immune checkpoint inhibitor, for patients with advanced, refractory squamous non-small-cell lung cancer (CheckMate 063): a phase 2, single-arm trial. Lancet Oncol 2015;16:257-65.

18. Brahmer J, Reckamp KL, Baas P, et al. Nivolumab versus Docetaxel in Advanced Squamous-Cell Non-Small-Cell Lung Cancer. N Engl J Med 2015;373:123-35.

19. Paz-Ares L, Luft A, Vicente D, et al. Pembrolizumab plus Chemotherapy for Squamous Non-Small-Cell Lung Cancer. N Engl J Med 2018;379:2040-51.

20. Jotte RM, Cappuzzo F, Vynnychenko I, et al. IMpower131: primary PFS and safety analysis of a randomized phase III study of atezolizumab + carboplatin + paclitaxel or nabpaclitaxel vs carboplatin + nab-paclitaxel as $1 \mathrm{~L}$ therapy in advanced squamous NSCLC. J Clin Oncol 2018;36 Suppl:LBA9000, abstract.

21. Liberati A, Altman DG, Tetzlaff J, et al. The PRISMA statement for reporting systematic reviews and metaanalyses of studies that evaluate health care interventions: explanation and elaboration. J Clin Epidemiol 2009;62:e1-34.

22. Fehrenbacher L, Spira A, Ballinger M, et al. Atezolizumab versus docetaxel for patients with previously treated non-small-cell lung cancer (POPLAR): a multicentre, open-label, phase 2 randomised controlled trial. Lancet 2016;387:1837-46.

23. Herbst RS, Baas P, Kim DW, et al. Pembrolizumab versus docetaxel for previously treated, PD-L1-positive, advanced non-small-cell lung cancer (KEYNOTE-010): a randomised controlled trial. Lancet 2016;387:1540-50.

24. Reck M, Rodriguez-Abreu D, Robinson AG, et al. Pembrolizumab versus Chemotherapy for PD-L1Positive Non-Small-Cell Lung Cancer. N Engl J Med 2016;375:1823-33.

25. Rittmeyer A, Barlesi F, Waterkamp D, et al. Atezolizumab versus docetaxel in patients with previously treated non-small-cell lung cancer (OAK): a phase 3, openlabel, multicentre randomised controlled trial. Lancet 2017;389:255-65.

26. Carbone DP, Reck M, Paz-Ares L, et al. First-Line Nivolumab in Stage IV or Recurrent Non-Small-Cell Lung Cancer. N Engl J Med 2017;376:2415-26.

27. Hellmann MD, Ciuleanu TE, Pluzanski A, et al. Nivolumab plus Ipilimumab in Lung Cancer with a High Tumor Mutational Burden. N Engl J Med 2018;378:2093-104.

28. Barlesi F, Vansteenkiste J, Spigel D, et al. Avelumab versus docetaxel in patients with platinum-treated advanced non-small-cell lung cancer (JAVELIN Lung 200): an open-label, randomised, phase 3 study. Lancet Oncol 2018;19:1468-79.

29. Wu YL, Lu S, Cheng Y, et al. Nivolumab Versus Docetaxel in a Predominantly Chinese Patient Population With Previously Treated Advanced NSCLC: CheckMate 078 Randomized Phase III Clinical Trial. J Thorac Oncol 2019;14:867-75.

30. Mok TSK, Wu YL, Kudaba I, et al. Final analysis of the phase III KEYNOTE-042 study: Pembrolizumab (Pembro) versus platinum-based chemotherapy (Chemo) as first-line therapy for patients (Pts) with PD-L1positive locally advanced/metastatic NSCLC. Ann Oncol 2019;30:Suppl:102O, abstract.

31. Brahmer JR, Pardoll DM. Immune checkpoint inhibitors: making immunotherapy a reality for the treatment of lung cancer. Cancer Immunol Res 2013;1:85-91.

32. Ishida M, Iwai Y, Tanaka Y, et al. Differential expression of PD-L1 and PD-L2, ligands for an inhibitory receptor PD- 
1 , in the cells of lymphohematopoietic tissues. Immunol Lett 2002;84:57-62.

33. Lin C, Chen X, Liu J, et al. Advances of PD-1/PDL1 signaling pathway in immune escape and treatment for non-small cell lung cancer. Zhongguo Fei Ai Za Zhi 2014;17:734-40.

34. Chen DS, Irving BA, Hodi FS. Molecular pathways: nextgeneration immunotherapy--inhibiting programmed death-ligand 1 and programmed death-1. Clin Cancer Res 2012;18:6580-7.

35. Matsumoto K, Fukuyama S, Eguchi-Tsuda M, et al. B7DC induced by IL-13 works as a feedback regulator in the effector phase of allergic asthma. Biochem Biophys Res Commun 2008;365:170-5.

36. Akbari O, Stock P, Singh AK, et al. PD-L1 and PD-L2 modulate airway inflammation and iNKT-cell-dependent airway hyperreactivity in opposing directions. Mucosal

Cite this article as: Li S, Zhang S, Liu J, Yang C, Zhang L, Cheng Y. The effect of PD-L1/PD-1 immunotherapy in the treatment of squamous non-small-cell lung cancer: a metaanalysis of randomized controlled clinical trials. J Thorac Dis 2019;11(11):4453-4463. doi: 10.21037/jtd.2019.11.12
Immunol 2010;3:81-91.

37. Gadgeel SM, et al. KEYNOTE-189: Updated OS and progression after the next line of therapy (PFS2) with pembrolizumab (pembro) plus chemo with pemetrexed and platinum vs placebo plus chemo for metastatic nonsquamous NSCLC. J Clin Oncol 2019;37:abstr 9013.

38. Zhou Y, Lin Z, Zhang X, et al. First-line treatment for patients with advanced non-small cell lung carcinoma and high PD-L1 expression: pembrolizumab or pembrolizumab plus chemotherapy. J Immunother Cancer 2019;7:120.

39. Mok TSK, Wu YL, Kudaba I, et al. Pembrolizumab versus chemotherapy for previously untreated, PD-L1expressing, locally advanced or metastatic non-small-cell lung cancer (KEYNOTE-042): a randomised, open-label, controlled, phase 3 trial. Lancet 2019;393:1819-30. 
Study

ID
$\%$

RR $(95 \% \mathrm{Cl}) \quad$ Weight

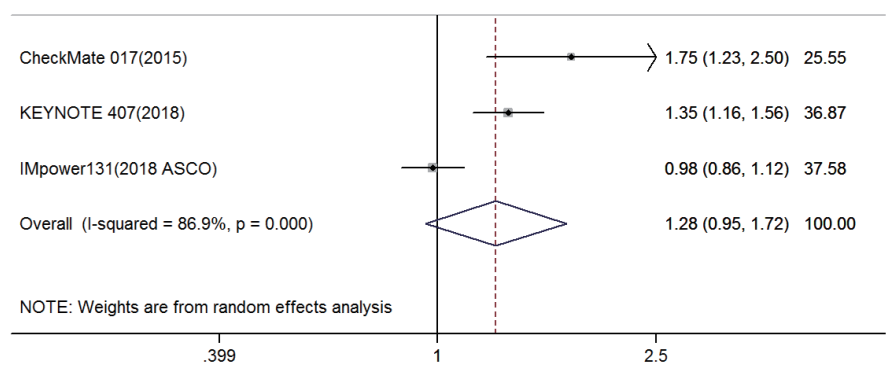

Figure S1 Forest plot of 1-y OS rate for comparison of PD-1/PD-L1 immunotherapy to chemotherapy in squamous non-small cell lung cancer. OS, overall survival.

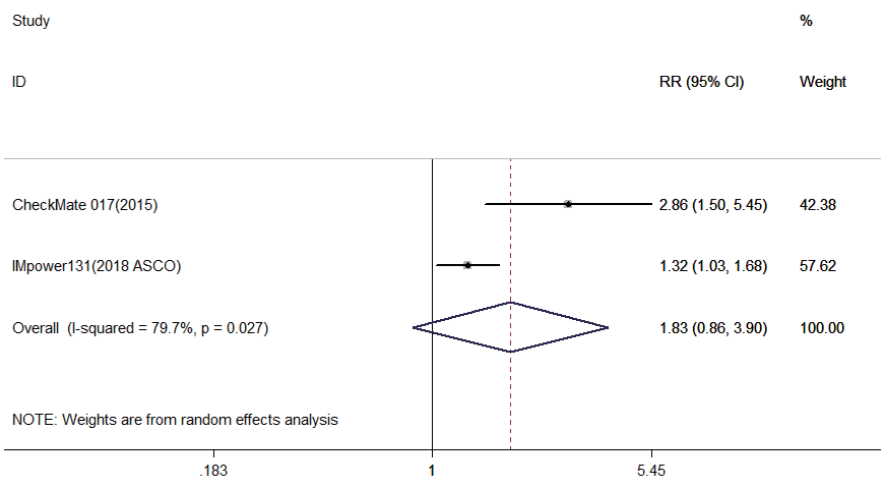

Figure S2 Forest plot of 2-y OS rate for comparison of PD-1/PD-L1 immunotherapy to chemotherapy in squamous non-small cell lung cancer. OS, overall survival.

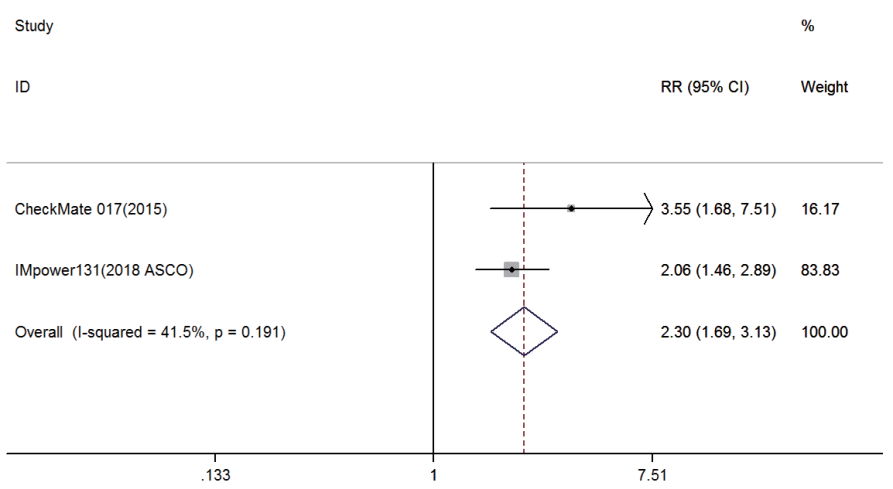

Figure S3 Forest plot of 1-y PFS rate for comparison of PD-1/PD-L1 immunotherapy to chemotherapy in squamous non-small cell lung cancer. PFS, progression-free survival. 
Study

ID
$\%$

$\operatorname{RR}(95 \% \mathrm{Cl}) \quad$ Weight

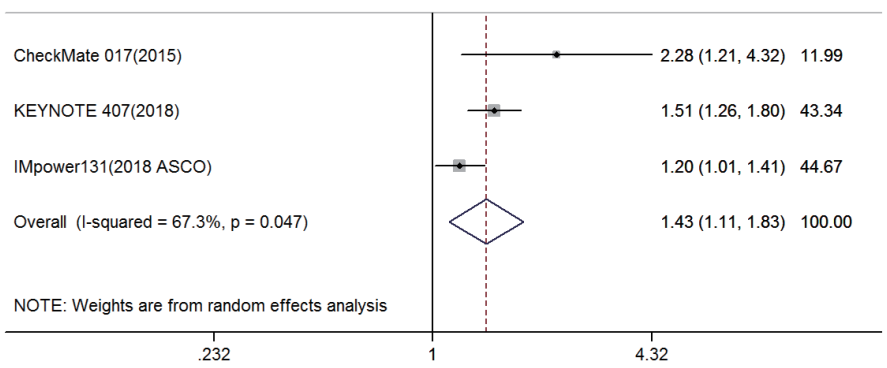

Figure S4 Forest plot of ORR for comparison of PD-1/PD-L1 immunotherapy to chemotherapy in squamous non-small cell lung cancer. ORR, objective response rate.

Study

ID
$\%$

$\mathrm{RR}(95 \% \mathrm{Cl}) \quad$ Weight

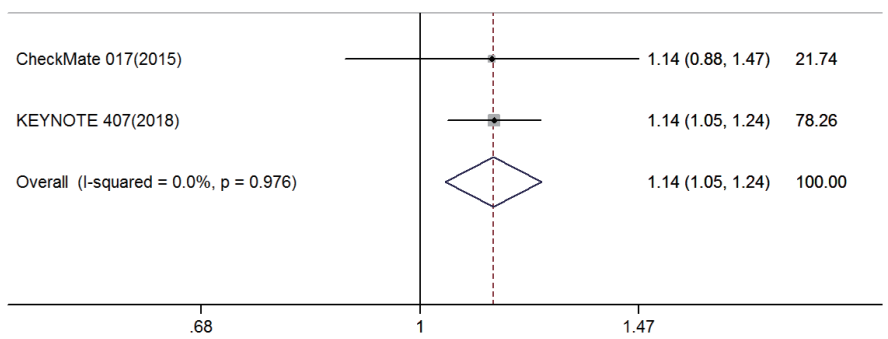

Figure S5 Forest plot of DCR for comparison of PD-1/PD-L1 immunotherapy to chemotherapy in squamous non-small cell lung cancer. DCR, disease control rate. 